\title{
Ground-based $K$-band detection of thermal emission from the exoplanet TrES-3b
}

\author{
E. J. W. de Mooij and I. A. G. Snellen
}

\author{
Leiden University, Postbus 9513, 2300 RA, Leiden, The Netherlands \\ e-mail: demooij@strw.leidenuniv.nl
}

Received 28 October 2008 / Accepted 28 November 2008

\section{ABSTRACT}

\begin{abstract}
Context. Secondary eclipse measurements of transiting extrasolar planets with the Spitzer Space Telescope have yielded several direct detections of thermal exoplanet light. Since Spitzer operates at wavelengths longward of $3.6 \mu \mathrm{m}$, arguably one of the most interesting parts of the planet spectrum (from 1 to $3 \mu \mathrm{m}$ ) is inaccessible with this satellite. This region is at the peak of the planet's spectral energy distribution and is also the regime where molecular absorption bands can significantly influence the measured emission.

Aims. So far, $2.2 \mu \mathrm{m} K$-band secondary eclipse measurements, which are possible from the ground, have not yet lead to secure detections. The aim of this paper is to measure the secondary eclipse of the very hot Jupiter TrES-3b in $K$-band, and in addition to observe its transit, to obtain an accurate planet radius in the near infrared.

Methods. We have used the william herschell telescope (WHT) to observe the secondary eclipse, and the united kingdom infrared telescope (UKIRT) to observe the transit of TrES-3b. Both observations involved significant defocusing of the telescope, aimed to produce high-cadence time series of several thousand frames at high efficiency, with the starlight spread out over many pixels.

Results. We detect the secondary eclipse of TrES-3b with a depth of $-0.241 \pm 0.043 \%(\sim 6 \sigma)$. This corresponds to a day-side brightness temperature of $\mathrm{T}_{\mathrm{B}}(2.2 \mu \mathrm{m})=2040 \pm 185 \mathrm{~K}$, which is consistent with current models of the physical properties of this planet's upper atmosphere. The centre of the eclipse seems slightly offset from phase $\phi=0.5$ by $\Delta \phi=-0.0042 \pm 0.0027$, which could indicate that the orbit of TrES-3b is non-circular. Analysis of the transit data shows that TrES-3b has a near-infrared radius of $1.338 \pm 0.016 R_{\mathrm{Jup}}$, showing no significant deviation from optical measurements.
\end{abstract}

Key words. techniques: photometric - stars: individual: TrES-3 - planetary systems

\section{Introduction}

During the secondary eclipse of an extrasolar planet (the moment the planet moves behind its host star), the contribution from the direct planet light can be measured. Until now such measurements have been the domain of the Spitzer Space Telescope, which have led to the first detections of thermal emission from extrasolar planets (Charbonneau et al. 2005; Deming et al. 2005). Until now a handful of planets has been observed at several wavelengths between $3.6 \mu \mathrm{m}$ and $24 \mu \mathrm{m}$, with one of the highlights so far being the measurement of the day/nightside temperature contrast of the exoplanet HD189733b (Knutson et al. 2007).

Unfortunately, a very interesting part of the broad spectral energy distribution of hot Jupiters between 1 and $3 \mu \mathrm{m}$ is shortward of the observing window of Spitzer. This region contains the overall peak of the planet's emission spectrum, and can also be strongly influenced by molecular bands (e.g. from water, methane and $\mathrm{CO}$ ) in the planets' upper atmosphere. However, ground-based observations, which could access this spectral region, have thus far proven very challenging. Several methods have been tried. Richardson et al. (2003) used a differential spectroscopic method, searching for the wavelength dependent shape of the planet spectrum, but no eclipse was detected. In addition, observations of the secondary eclipse of TrES-1b in $L$-band with the NIRI spectrograph on Gemini North by Knutson et al. (2007) only resulted in an upper limit.

Photometric attempts to detect the secondary eclipse of extrasolar planets have been presented by Snellen (2005) and Snellen \& Covino (2007). Two partial eclipses of HD 209458b were observed in $K$-band by Snellen (2005), while the telescope was significantly defocused to avoid saturation of the array by the bright star. Although milli-magnitude precisions were reached, nothing was detected, probably because of the lack of baseline on both sides of the eclipse (Snellen 2005). Subsequently, OGLE-TR-113b was observed (Snellen \& Covino 2007), which has the great advantage that it has several other stars in its field. It was shown that randomly offsetting the telescope randomises the photometric errors down to $0.1-0.2 \%$ per hour. It resulted in a tentative detection of its secondary eclipse of $0.17 \pm 0.05 \%$.

In this letter we present our results for $K$-band photometry of the transit and secondary eclipse of the planet TrES-3b (O'Donovan et al. 2007). This planet is significantly more suitable for secondary eclipse photometry than previous targets, since it is in a very close orbit $(P \sim 1.31$ days $)$ and its radius is significantly inflated $\left(R \sim 1.3 R_{\mathrm{Jup}}\right)$. Furthermore there is a nearby reference star, which can be used for differential photometry. In Sect. 2 we present our observations and data reduction. In Sect. 3 we present and discuss our results.

\section{Observations, data reduction and analysis}

\subsection{The transit of TrES-3b}

We have observed the transit of the exoplanet TrES-3b with the United Kingdom Infrared Telescope (UKIRT) using its Fast Track Imager (UFTI; Roche et al. 2003) on June 20, 2008. 


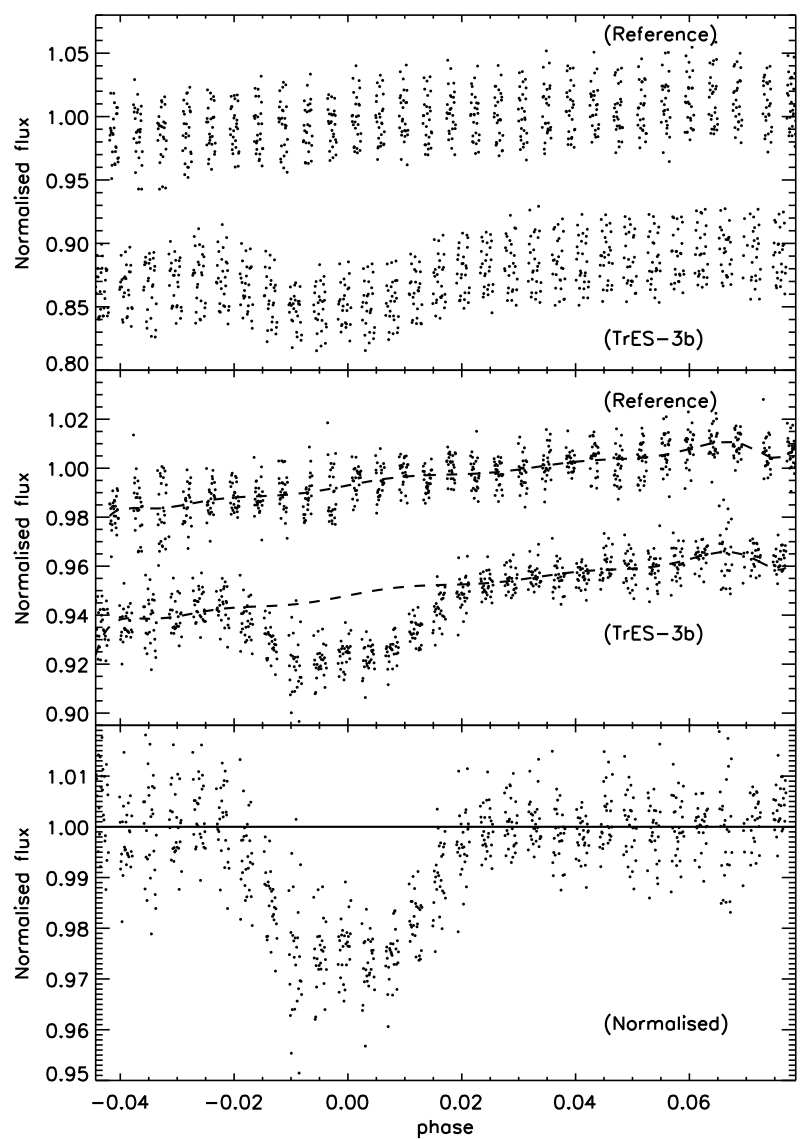

Fig. 1. $K$-band lightcurves from the UKIRT transit observations. The top panel shows the lightcurve of the reference star and TrES-3b before correcting for the positional dependence of the flux. The middle panel shows the fluxes of both stars corrected for this effect. The dashed line is a polynomial fit to the flux of the reference star. The bottom panel shows the final corrected unbinned lightcurve of TrES-3b.

The observations, carried out in queue scheduling, lasted for almost $4 \mathrm{~h}$, starting $\sim 0.6 \mathrm{~h}$ before ingress and ending $\sim 1.7 \mathrm{~h}$ after egress. The field of view of UFTI is $93 \times 93$ arcsec, with a pixel scale of $0.091 \mathrm{arcsec} / \mathrm{pixel}$. Since the distance between TrES-3 $(K=10.61)$ and the nearby reference star (2MASS $\mathrm{J} 175225.15+373422.1, K \sim 9.77)$ is $\sim 4$ arcmin, we were forced to alternate observations between the target and the reference, in order to correct for time dependent atmospheric and instrumental effects. To reduce overheads from detector read-out, the detector was windowed to a single quadrant $(512 \times 512$ pixels, $46.6 \times 46.6$ arcsec) which increased the cycle speed. During each sequence we repeated a nine point dither pattern three times before switching to the other star. The exposure time was $5 \mathrm{~s}$ for the TrES-3, and $2 \mathrm{~s}$ for the reference star. With a typical overhead of $5 \mathrm{~s}$ per frame, the time required to complete an entire target-reference sequence was $\sim 8 \mathrm{mn}$.

The telescope was significantly defocused to 1) reduce intrapixel variations; 2) minimise the influence of flatfield inaccuracies; and 3) prevent the count levels from reaching the nonlinear range of the detector. Due to the fact that the telescope optics had not yet been realigned after the recent switch to the Cassegrain focus, the defocused telescope produced a significantly asymmetric PSF, which could be covered by a circular aperture of $\sim 40$ pixels $\left(\sim 4^{\prime \prime}\right)$ in radius.

The data was flatfielded using a sky flat constructed from a series of frames taken just before the start of the observations of

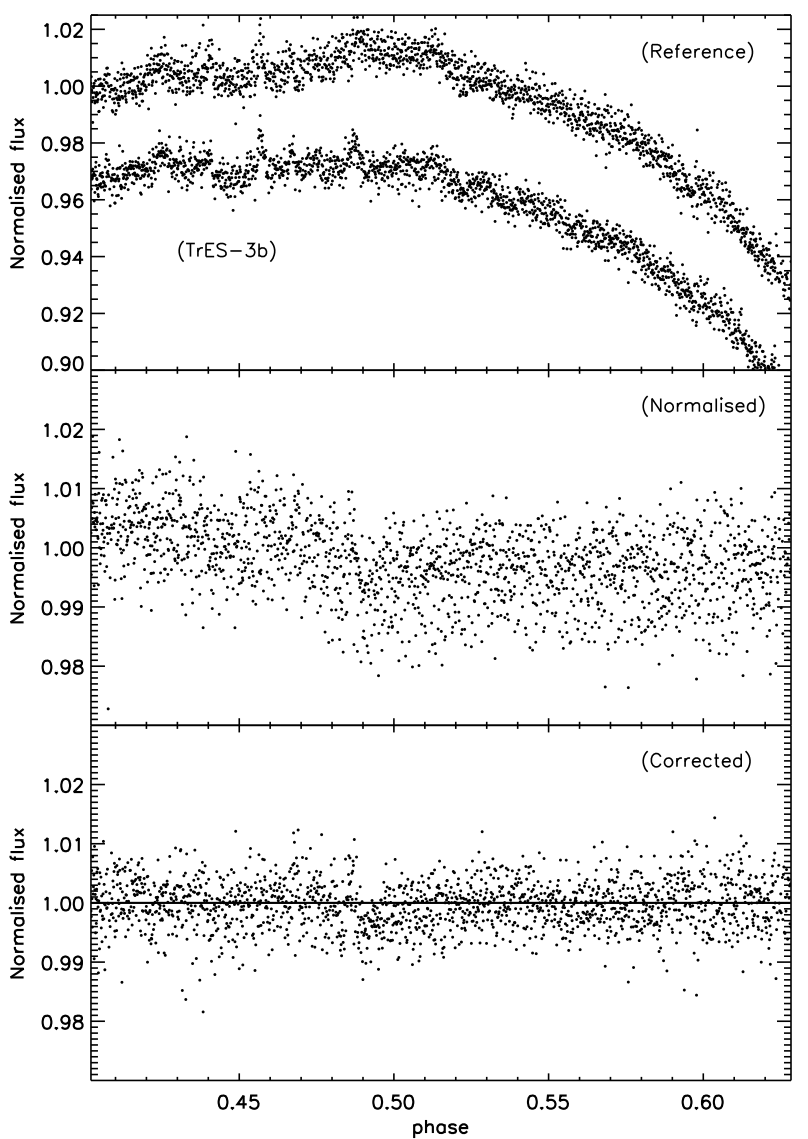

Fig. 2. $K$-band lightcurves from the WHT secondary eclipse observations. The top panel shows the raw lightcurve for the reference star and TrES-3b. The middle panel shows the lightcurve of TrES-3b divided by that of the reference star. The bottom panel shows the final, fully corrected, lightcurve.

TrES-3. No dark subtraction was performed, because any darkcurrent was seen as a contribution to the sky background and removed together with the sky. The positions of the hot and cold pixels were determined from both the flat field and separate dark frames. These pixels were replaced by the values of 3rd order polynomial surfaces fitted to the $7 \times 7$ pixels surrounding these pixels.

To determine the sky background, first all stars in the field were masked using circular masks of 30 pixels. A median sky value for the masked image was calculated and subtracted in order to remove temporal fluctuations in the background. The final sky level was determined by averaging the 27 masked frames from one cycle, and removed from the images.

Subsequently, aperture photometry was performed using the APER procedure from the IDL Astronomy User's Library ${ }^{1}$ with an aperture radius of 42 pixels. Any possible residual sky fluctuations were corrected for by measuring the background value in a 42 to 70 pixel annulus around the object.

The results from the aperture photometry can be found in the top panel of Fig. 1. Both the flux from the target and the reference star was found to be a function of dither position, therefore the data were normalised for each dither position separately. These corrected lightcurves are shown in the middle panel of Fig. 1. To remove time dependent effects, we fitted a high order polynomial to the binned lightcurve of the reference star. This fit

${ }^{1}$ http://idlastro.gsfc.nasa.gov 
was used to correct the lightcurve of TrES-3. The final unbinned lightcurve for TrES-3 is shown in the bottom panel of Fig. 1.

\subsection{The secondary eclipse of TrES-3b}

On July 3-4, 2008 the secondary eclipse of TrES-3 was observed with the Long-slit Intermediate Resolution Infrared Spectrograph (LIRIS; Acosta-Pulido et al. 2002) at the William Herschell Telescope (WHT) on La Palma, with the observations lasting for the entire night, starting about $\sim 3 \mathrm{~h}$ before, and ending $\sim 4 \mathrm{~h}$ after the expected centre of the secondary eclipse (at 1:31 UT on July 4th). We used the full $1024 \times 1024$ pixel array for these observations, which combined with the pixel size of LIRIS of $0.25^{\prime \prime} /$ pixel, yields a field of view of $256 \times 256$ arcsec. This larger field of view enabled us to place both TrES-3 and the reference star simultaneously on the detector, making these observations $\sim 80 \%$ more efficient relative to those obtained with UKIRT. We used a 9 point dither pattern, including small random offsets from the nominal positions of each dither point. We used an exposure time of $7.5 \mathrm{~s}$, with typical overheads of $7 \mathrm{~s}$ per frame.

As for the UKIRT observations, we defocused the telescope, which resulted in a ringshaped PSF with a radius of about 4 pixels.

Since the LIRIS detector is non linear at high count levels, we applied the following empirical non-linearity correction to the data before flat fielding:

$F_{\text {true }}=F_{\text {meas }} \cdot \frac{1}{1-c \cdot F_{\text {meas }}^{6}}$

with $F_{\text {true }}$ the flux after correction, $F_{\text {meas }}$ the measured flux and $c=6 \times 10^{-29}$ a constant describing the strength of the correction term. The value of $c$ was determined by minimising the noise of the reference star over the entire night, and comparing this value to non-linearity measurements taken by the LIRIS team ${ }^{2}$.

All frames were corrected for crosstalk between the quadrants, which was found to be present in a co-add of all the reduced science frames at a level of $10^{-5}$ of the total flux along a row of a quadrant. This correction was done on a row by row basis. A flat field was created from a series of domeflats taken with the domelight both on and off. These were subtracted from each other (to eliminate structure from the emission by the dome and telescope) and averaged.

The small number of hot and cold pixels in the image were replaced by a median of the neighbouring pixels in a $3 \times 3$ grid. The sky was determined from the combination of the 9 images in each dither sequence, for which all the stars were masked out. We used a circular aperture with a radius of 13 pixels to determine the stellar flux and an annulus of 30 to 90 pixels to determine the level of possible residual flux in the background after sky-subtraction.

The individual raw lightcurves of TrES-3b and the reference star are plotted in the top panel of Fig. 2. Before further analysis we divided the lightcurve of TrES-3 by that of the reference star, shown in the middle panel of Fig. 2. The relative fluxes of the two stars are correlated with their positions on the detector. These effects are corrected for by fitting linear functions between the $x, y$-positions and the relative flux for data outside of the expected eclipse. These fits are then applied to the whole dataset. In a similar way a small dependence on airmass was removed.

\footnotetext{
2 http://www.ing.iac.es/Astronomy/instruments/liris/ liris_detector.html
}

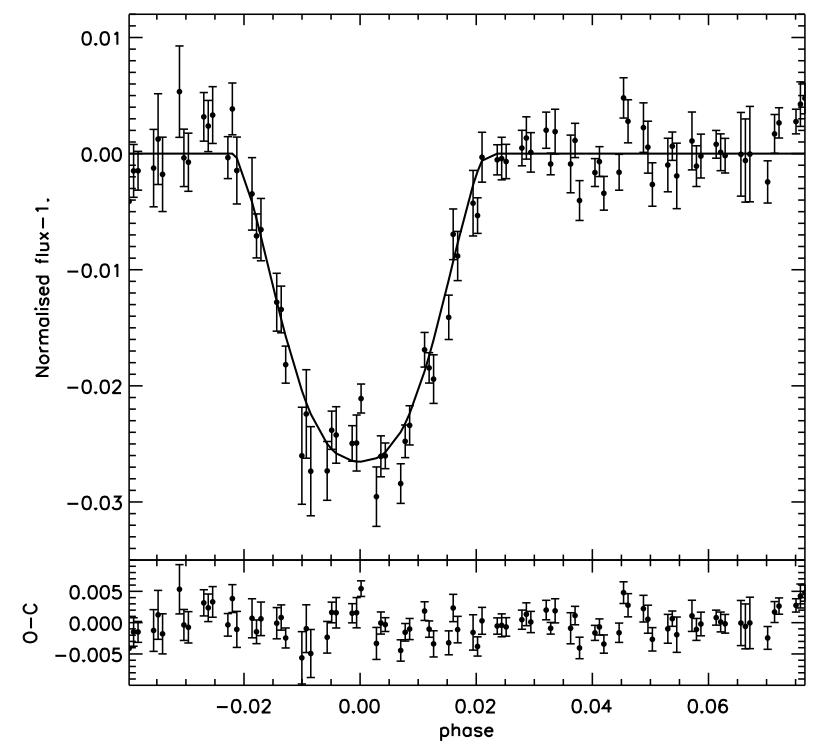

Fig. 3. The 9-point binned lightcurve of the transit of TrES-3b. The solid line shows the best fitting model with $R_{\mathrm{P}}=1.338 \pm 0.016 R_{\text {Jup }}$. The bottom panel shows the residuals after subtracting this model fit.

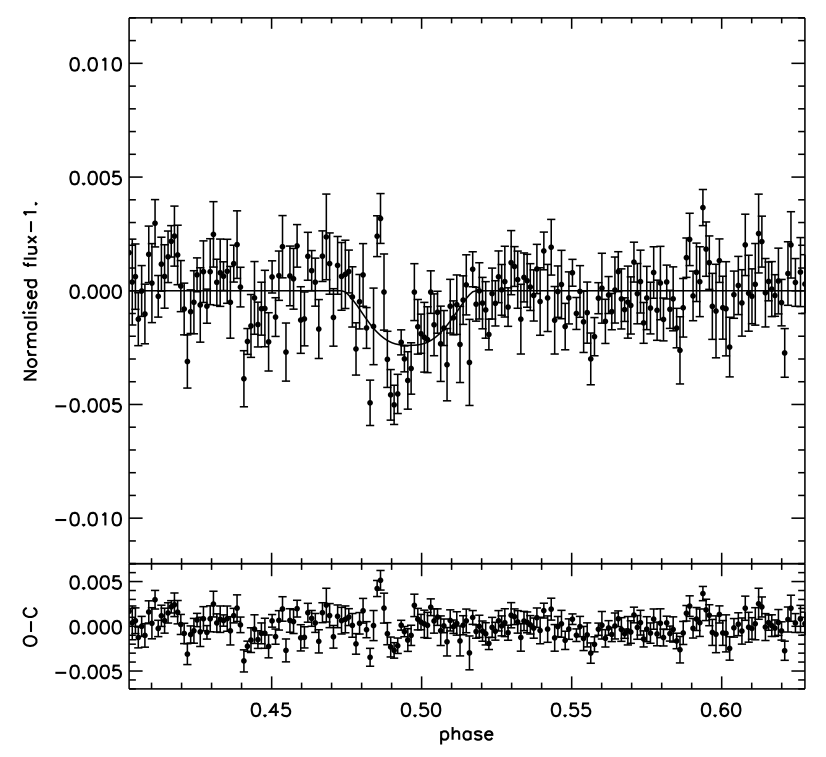

Fig. 4. Final, 9-point binned lightcurve of the secondary eclipse. The solid line is the best fitting model with $\Delta F=-0.241 \pm 0.043 \%$ and an offset from phase 0.5 of $\Delta \phi=-0.0041 \pm 0.0018$. The bottom panel shows the residuals after subtracting the best fitting model.

The corrected unbinned lightcurve of TrES-3b can be found in the lower panel of Fig. 2.

\section{Results and discussion}

\subsection{The transit of TrES-3b}

The final transit lightcurve binned per 9-point dither cycle is shown in Fig. 3. The rms-noise level out of transit is 0.0060 , while the theoretical noise level for these observations is 0.0029 , which is actually dominated by the detector's readnoise of $26 \mathrm{e}^{-} /$pixel, due to the large aperture used.

Since a dependence of the transit depth on wavelength can reveal characteristics of the planet atmosphere, we fitted the radius of TrES-3b, keeping the parameters of the host star and 
the planet's orbit (mean stellar density, orbital inclination and ephemeris) fixed with respect to the values of Sozzetti et al. (2008). For the limb darkening we used parameters from Claret (2000), assuming a temperature of $T=5750 \mathrm{~K}$ for the host star of TrES-3b, a metalicity of $\log [\mathrm{Fe} / \mathrm{H}]=-0.2$ and a surface gravity of $\log (\mathrm{g})=4.5$. The transit was modelled using the IDL procedures from Mandel \& Agol (2002), resulting in a radius of $1.338 \pm 0.013 R_{\text {Jup }}$ at $\chi^{2} / v=1.58$. The reduced $\chi^{2}$ is significantly larger than unity. Since we fitted to the binned lightcurve, this indicates that the noise does not scale with $\sqrt{N}$, meaning that we have an additional, unknown, source of correlated noise. To obtain a better estimate of the uncertainty in the planet radius, we forced $\chi^{2} / v=1$ by scaling up our errors in the binned lightcurve uniformly. In this way we obtain $R_{\mathrm{P}}=1.338 \pm 0.016 R_{\text {Jup }}$. The $K$-band radius shows no significant deviation from the optical radius of $1.336_{-0.037}^{+0.031} R_{\mathrm{Jup}}$, as measured by Sozzetti et al. (2008). The uncertainty in our $K$-band radius appears smaller, but this is solely due to the fact that we keep the parameters for the host star and the planet's orbit fixed.

We show that we can obtain the $K$-band radius of an exoplanet at a precision of $\sim 1 \%$. This could be further improved by observing the reference star and target at the same time both because of the increase in the number of frames during transit and because rapid atmospheric fluctuations can be corrected for.

\subsection{The secondary eclipse of TrES-3b}

The fully corrected lightcurve binned by one 9 point dither cycle is shown in Fig. 4. Before binning, and after subtracting the best fitting model (see below), we clipped the lightcurve at \pm 0.01 , removing 43 of the 1800 points $(2.3 \%)$, of which 3 points $(0.9 \%)$ during the eclipse. The binned lightcurve was fitted using the Mandel \& Agol (2002) procedures, with all the parameters fixed to the transit model, except the centre and the depth of the eclipse (of course without limb darkening).

The best fitted lightcurve is over-plotted in the top panel of Fig. 4, with the bottom panel showing the residuals. The out of eclipse rms noise is 0.0038 , while the expectation from theoretical noise statistics is 0.0015 . The dominant source of noise in these noise calculations is the sky contribution. The total flux from the sky within the aperture is, even for the brighter reference star, $\sim 50 \%$ higher than the stellar flux.

The fitted depth of the secondary eclipse is $\Delta F=-0.241 \pm$ $0.021 \%$, with a reduced chi-squared of $\chi^{2} / v=1.72$. As for the transit data, the reduced $\chi^{2}$ for the fit to the binned lightcurve is greater than unity, indicating that we have some extra systematic noise. We also find a timing difference in phase of $\Delta \phi=$ $-0.0041 \pm 0.0013$ (at $\chi^{2} / v=1.72$ ). The offset in the timing is intriguing, because it may indicate that the orbit of TrES-3b is not circular but slightly eccentric, with ecos $\omega=-0.0066 \pm 0.0021$. Although the formal significance of the offset is $\sim 3 \sigma$, the residual systematic noise in the lightcurve decreases its significance, in particular due to several points around $\phi=0.49$. We also refitted the data with the centre of the eclipse fixed to $\phi=0.5$, and obtained in this case a depth of $-0.233 \pm 0.020$ at $\chi^{2} / v=1.77$.

To test the influence of the more deviated points around $\phi=0.49$, we repeated the fit while excluding them. The depth subsequently becomes $-0.190_{-0.037}^{+0.034}$, with an offset in phase of $\Delta \phi=-0.0036_{-0.0020}^{+0.0022}\left(\chi^{2} / v=1.39\right)$. Although the significance of the fit is reduced, we still detect the secondary eclipse at a $6 \sigma$-level.

Because it is clear that red noise dominates the uncertainties in our measurements, we tried to characterise the uncertainty using the "residual permutation" bootstrap method as used by Winn et al. (2008). The best fitting model was subtracted from the data, after which the residuals were shifted between 1 and 1800 points and added to the model light curve. These light curves were refitted for each individual shift. The resulting distributions of parameter values represent better estimates of the real uncertainties in the data. With this analysis we find an uncertainty of $4.3 \times 10^{-4}$ in the eclipse depth and $2.7 \times 10^{-3}$ for the offset in phase, which we use in the remainder of the paper.

Assuming a blackbody model for the thermal emission spectrum of TrES-3b, we can estimate the day-side brightness temperature in the $K$-band. For the stellar temperature, we use $T_{\text {eff }}=$ $5650 \pm 75 \mathrm{~K}$ (Sozzetti et al. 2008). From the fit to the eclipse depth using the full lightcurve we obtain $T_{\mathrm{B}}=2040 \pm 185 \mathrm{~K}$ ( $90 \%$ confidence interval).

Due to the high incident stellar flux, Fortney et al. (2008) classify this planet as belonging to the pM class of planets. This class is expected to have an inversion layer in their upper atmospheres and to show a large day/night contrast. Comparing the depth of the secondary eclipse with their model (their Fig. 14), we see that our results are indeed consistent with this model. However, since we have measured the brightness temperature of TrES-3b at only single wavelength, Spitzer measurements at longer wavelengths are needed to confirm the presence of an inversion layer and thus classify TrES-3b as a pM planet.

\section{Conclusions}

We have detected the secondary eclipse of the exoplanet TrES$3 \mathrm{~b}$ in $K$-band at a level of $-0.241 \pm 0.043 \%$, indicating a brightness temperature of $T_{B}(2.2 \mu \mathrm{m})=2040 \pm 185 \mathrm{~K}$. The eclipse timing shows a small offset from $\phi=0.5$, which would imply a non-circular orbit, but this needs to be confirmed by Spitzer observations. Also the radius of TrES-3b is determined in $K$-band to be $1.338 \pm 0.016 R_{\text {Jup }}$, showing no deviations from optical measurements.

Acknowledgements. We are grateful to the UKIRT observers and the staff of both the UKIRT and WHT telescopes. The United Kingdom Infrared Telescope is operated by the Joint Astronomy Centre on behalf of the Science and Technology Facilities Council of the UK. The William Herschel Telescope is operated on the island of La Palma by the Isaac Newton Group in the Spanish Observatorio del Roque de los Muchachos of the Instituto de Astrofísica de Canarias.

\section{References}

Acosta-Pulido, J., Ballesteros, E., Barreto, M., et al. 2002, The Newsletter of the Isaac Newton Group of Telescopes, 6, 22

Charbonneau, D., Allen, L. E., Megeath, S. T., et al. 2005, ApJ, 626, 523

Claret, A. 2000, A\&A, 363, 1081

Deming, D., Seager, S., Richardson, L. J., \& Harrington, J. 2005, Nature, 434, 740

Fortney, J. J., Lodders, K., Marley, M. S., \& Freedman, R. S. 2008, ApJ, 678, 1419

Knutson, H. A., Charbonneau, D., Allen, L. E., et al. 2007, Nature, 447, 183

Mandel, K., \& Agol, E. 2002, ApJ, 580, L171

O’Donovan, F. T., Charbonneau, D., Bakos, G. Á., et al. 2007, ApJ, 663, L37 Richardson, L. J., Deming, D., \& Seager, S. 2003, ApJ, 597, 581

Roche, P. F., Lucas, P. W., Mackay, C. D., et al. 2003, in Proc. SPIE 4841, ed. M. Iye, \& A. F. M. Moorwood, 901

Snellen, I. A. G. 2005, MNRAS, 363, 211

Snellen, I. A. G., \& Covino, E. 2007, MNRAS, 375, 307

Sozzetti, A., Torres, G., Charbonneau, D., et al. 2008 [arXiv: 0809.4589v1]

Winn, J. N., Holman, M. J., Henry, G. W., et al. 2008 [arXiv:0810.4725v1] 